\title{
An Overview of the Studies on the Generation of Typical Middle Construction
}

\author{
Xiaohua Zhai \\ Shanxi Vocational College Of Tourism, Taiyuan 030031, China

\begin{abstract}
This paper mainly takes an overview of previous studies on the generation of middle constructions. First is the introduction of the research background of the generation of middle constructions. Then the author describes the main syntactic features of typical middle constructions, followed by an overview of previous studies on this construction It can be seen that each study has its advantages and disadvantages. Through this review, the author advise to study the middle construction from different aspects because of its complexity. Through this review, the author hopes to find some space for future study.
\end{abstract}

Keywords: Middle Construction; Generation; Syntactic Feature

COPYRIGHT

Copyright (C) 2019 by author(s) and Frontier Scientific Publishing. This work is licensed under the Creative Commons

Attribution-NonCommercial 4.0 International License (CC BY-NC 4.0). http://creativecommons.org/licenses/

\section{Introduction}

The following sentences belong to middle constructions frequently cited by researchers:

(1) Bureaucrats bribe easily.

(2) The wall paints easily.

The above examples all have "Patient" as their subject, so they are called "Patient middle construction." In addition, researchers also find "adjunct middle construction". Adjunct middle construction can be fulfilled by the components expressing locations and tools, for example:

(3) The blackboard writes smoothly.

(4) The pen writes well.

(3), (4) have similar syntactic and semantic representations to (1) and (2), the non-agent component occupying the subject position. The sentences expresses certain properties or characteristics of the grammatical subject and they must contain a adverb or an adjective at the end of the sentence as the modifier. We agree with the above researchers that the structures like (3) and (4) should be included in the scope of middle construction. This paper only deals with the above two types of typical Chinese and English intermediate constructions, namely patient middle construction like (1) and (2) and the non-patient middle construction like (3) and (4), where (3) has the subject of location and (4) of tool.

Recently, middle constructions have become one of the hot topics in the study of generative grammar. Many scholars have engaged in heated discussions around the characteristics of middle constructions and their generation mechanism. The focus of the discussion is the essential characteristics of middle constructions. That is, whether the process of generating middle construction is lexical or syntactic. From previous research, in the nature of the middle construction generation process, two opposing views can be summarized. Some researchers insist that the construction of middle constructions rely entirely on lexical means, which does not involve syntactic operations such as movement, and is purely a pre-syntactic operation. Because the logical subject of the middle not enter the stage of syntactic operation, 
there is no way to talk about its syntactic status. Another view insists on the idea of generation through the syntactic approach, which means that the structure of the middle construction requires the demotion of the external argument and the syntactic promotion of the internal arguments The logical subject of the middle exists at the syntactic level and has syntactic functions. This paper first introduces the main features of the typical middle construction, gives a preliminary overview of the research on the generation of the middle sentences and briefly states some thoughts of the author. Finally, it tries to see the prospects for future research.

\section{The main syntactic features of typical middle constructions}

The grammatical subject of the middle construction is the patient or the adjunct argument of the verb Most researchers consider that the verb has an implicit agent.

The middle construction has a typical general. Keyser and Roeper pointe out that the function of the middle construction is to refer generally and make a statement. Therefore, it should not be used to describe a specific event at a particular time. The middle, like the ordinary verbs, is not absolutely not used for the progressive aspect, but it does not express the event at this time. If used with progressive tense, it means that the state is changing with time.

Middles generally require modification of trait adverbs such as "easily", otherwise sentences are usually grammatically unacceptable. Fagan argues that middle constructions are not used to report events, but rather to state that an object has a particular property. Therefore, middles require trait modifiers such as "easily" to describe the properties of the grammatical subject of the sentence.

Researchers also believe that the middle sentences often have modal meanings, but such middle sentence (usually containing modal verbs) are not typical middle sentences, and are not included in the scope of this paper.

\section{Research on the generation mechanism of typical middle construction}

\subsection{Lexical approach}

Lexical Approach considers that the middles become intransitive verbs in the lexicon, and the external argument is suppressed before entering the syntactic operation, and the internal argument is projected as the subject. Lexical Approach needs to preset complicated lexicon operation rules, which increases the burden of the lexicon and is not conducive to acquisition. Fagan believes that the middle construction does not allow preposition stranding, so the generation of the structure does not involve NP movement. The middle construction is not generated by lexical means but by syntactic means. Fagan emphasizes that the generalization of meaning is the key to the construction of the middle construction. The middle verb usually uses present tense. Keyser \& Roeper attribute this to their general meaning. However, the difference between the middle construction and other generalized propositions is that it does not require its own surface grammar subject to express general meaning. In fact, it is the implicit agent of the middle verb that is usually understood to have general meaning. Levin points out that the middle verb has an implicit argument modified by a generalized quantifier, which can be understood as "people in general".

\subsection{Syntactic approach}

\subsubsection{Passivization analysis}

Keyser and Roeper argue that the generation of middle constructions undergo syntactic operations similar to passivization. In the passive structure, the passive verb is derived from the active two-place predicate by adding the past participle affix to it. The middle verb is also derived from the corresponding transitive verb, but not by adding the affix, but through a word-formation rule. This rule, like passive affixes, absorbs the theta role of the logical subject, while suppressing the case assignment to the logical object. Driven by the requirement of case, the logical object raises to the subject position to obtain nominative.

Stroik propose that the generation of the middle construction involves NP movement, and two correlation 
operations are required: the demotion of the external argument and the promotion of the internal argument. Obviously, this derivation process is similar to the derivation of passive sentences. Dai Manchun, based on the research of Stroik, proposes an analysis model of the middle construction with patient subjects in English and Chinese. He believes that the middle verb is similar to the passive verb, whose external argument is suppressed, and the ability assign accusative is also suppressed, and the internal argument obtains the features of nominative. In his opinion, the middle verb has a complete argument structure syntactically, and there exists a middle morpheme similar to the passive suffix, mainly used to cancel accusative assignment.

Zhou Xiaoyan and Gao Teng also propose an analysis model of the middle constructions with patient subjects in English and Chinese. They believe that the D- structure of the middle sentence "这车开起来挺容易" has the Dstructure " 人们开这车挺容易". " 起来" in the sentence is a verb of ability. It is precisely because of the insertion of " 起来" that leads to the lack of theta role in the position of the subject, which prevents the verb from assigning case to the patient, Under the requirement of case, the object moves to the subject position in the sentence. They believe that this inserted component exists as an implicit form in English.

\subsubsection{Voice projection and transitivity projection}

Collins sets VoiceP obove vP, providing Smuggling Analysis for the internal argument of the verb. Bowers insists setting up TrP under vP to check the accusative but this does not give license to arguments. Separating the ability to introduce external argument with that of checking accusative, Bowers explains the situation where the internal argument moves to the position of grammatical subject. He also uses the parameterization of transitive features to explain the inter-language differences of the middle construction. However, adding functional categories for the syntactic derivation of passive and middle sentences lacks of conceptual necessity.

\subsubsection{Analysis based on applicative hypothesis and phase theory}

Based on of previous studies, He Xiaowei (2012) proposes the generation model of two kinds of typical middle constructions in Chinese and English in the framework of phase theory. He considers that (1) the modifier at the end of the sentence of the typical middle construction acts as the non-core complement, which is an essential component of the construction. The component is closely related to the middle verb, and the verb $\mathrm{V}$ is first merged with this component, and the generated $\mathrm{V}^{\prime}$ is responsible for the permission of the theta roles of related noun phrases in the structure. (2) There exists a middle morpheme in the middle construction, which is represented as "起 来 " in Chinese, as an empty category in English. The morpheme is attached to the light verb v. The vP formed by the light verb v does not form a phase because it does not have an external argument, and is not restricted by PIC in syntactic derivation. (3) The location and tool arguments in the non-patient middle construction are non-core arguments, and are introduced by the high applicative head H-APPL. On the basis of the above assumptions, the derivation of the two types of typical Chinese and English structures as well as related theoretical issues have been uniformly explained.

In this analysis, the vP containing the middle morpheme does not form a phase because it does not have an external argument, and thus it is not restricted by PIC. The component $\mathrm{T}$ pit of vP can operate on the components in the vP, making the noun phrases expressing patient, location and tool het the features of nominative and move to the subject position of the sentence. The modifier at the end of the sentence serves as the non-core complement of the sentence, and merges with the verb and other components to jointly permit the theta roles of the relevant noun components. It can be seen that the modifiers at the end of the sentence and the middle morpheme play a significant role in the syntactic derivation of the structure.

\subsubsection{The analysis of stativization process}

Han Jingquan $(2003,2004)$ refers to the transformation of verbs from expressing behavior to expressing state as "stativization" of verbs. He considers that it is this kind of stativization of verbs constitutes the basis of the generation of middle constructions. Stativization first changes the argument structure of transitive verbs. The same verb, taking 
"paint" as an example, when used as a transitive verb and as an middle verb, has the argument structures (a) and (b) respectively:

(a) paint ( $\mathrm{x}(\mathrm{y}))$

Agent Theme

(b) paint ( $\mathrm{x}-\mathrm{O} /(\mathrm{y}))$

Agent Theme

The stativization of the middle verbs is actually the transformation from action verbs to state verbs, or more precisely, from the agency action description to the statement of the nature of an event. After the transformation, the original logical agent subject of the verb is suppressed, and the verb loses the ability to assign case to the logical object. In order to meet the needs of the extended projection principle and the case feature checking, through syntactic movement of the logical object, it gets out of the domain and reaches the position of grammatical subject, generating middle constructions. In essence, stativization of the middle verbs is the formation of a new word based on the rules of word formation. The composition of new word items sometimes changes the original argument structure.

\section{The advantages and disadvantages of each analysis}

As for lexical approach, both in the analysis of Fagan and in that of Achema \& Schoorlemmer, the referential semantic values of the logical subject of the middle verbs are pre-set to be general meaning and this general meaning is used as the basis of middle constructions. However, in some contexts, especially when the verbs are used with progressive aspect or past tense, the semantic values of the implicit logical subject can be specific, which contradicts their presupposition.

The voice projection and transitivity projection analysis also explain the reason why the inernal argument moves to the subject position, but adding a new function category for the syntactic derivation of middle constructions lacks of conceptual necessity (Wang Heyu \& Wen Bingli, 2014). The addition of a new concept just for the interpretation of a certain linguistic phenomenon must also not conform to the orientation of universal grammar theories.

Phase theory and applicative hypothesis analysis can provide a unified explanation for middle verbs with an agent subject and those with a non-agent subject, and at the same time make the necessity of the existence of the modifiers at the end of the sentence very clear, but Zhang Zhiyi and Ni Chuanbin (2014) pointe out that He Xiaowei's (2012) analysis might not be very convincing because in fact, English is essentially a low-applicative language, so it cannot be said that English has high-applicative structures because of the raising and movement of the arguments. There is no applicative in this type of middle constructions. From this point of view, the analysis is not invulnerable.

The advantage of the "stativization" process analysis is that it can incorporate the various features of middle constructions as a whole into a unified explanation. The analysis holds that the stativization of the verb is the essential feature of the middle construction, and other features are derived from this one.

In addition, as Shi Dingxu (2019) said, if the methods and experiments of neural ience can be effectively applied and the processing of middle constructions in the brain can be monitored to find the specific EEG component of the structure processing, such evidence should also promote our understanding of the generation of middle constructions. Or it can be said that the study of generative grammar and the evidence of neural science and technology complete one another.

\section{Reflection, conclusion and prospect}

Dai Manchun's (2001) analysis agrees with the passivization analysis. He believes that the external argument of the middle construction is not completely suppressed, and thus can be introduced by the complementizer "for". Middle verbs have a complete argument structure. However, Han Jingquan (2003) and Zhang Zhiyi and Ni Chuanbin (2014) believe that the external argument in this kind of structures is completely suppressed, and thus such arguments 
have no syntactic status. However, the agent introduced by the preposition "by" in the passive sentence is an external argument that has undergone demotion and has its syntactic status. The author of this paper partially agrees with the latter point of view that the middle construction does not carry an external argument, in that this can be structurally justified by Burzio's Generalization, the middle verbs have undergone "case adsorption" and cannot assign case to the internal argument, and so they have no external argument (Haegeman, 1994). But at the same time, the author of this paper also considers that it is still unclear whether the difference between the agent introduced by the preposition "by" in the passive sentence and the agent introduced by the complementizer "for" in middle constructions is so great. After all, both of them can introduce the semantic role of agent. They are both at the end of the sentence in the linear structure of the sentences and act as adjuncts. Whether it is based on the principle of "uniformity" (Wen Binli, 2002) or based on psychological reality, the two noun phrases seem to be less likely to have such a great difference in syntactically.

In summary, it can be seen that there are advantages and disadvantages for different analysis of middle constructions. Although researchers have reached some consensus, there are still many and even essential divergences. Due to the complexity of its construction, middle construction involves many aspects such as syntax, semantics and pragmatics, so further research is needed.

In this structure, the interaction syntactic-semantic interaction is quite obvious, so research on the permissions of the structure from the semantic and cognitive level may also provide us with new thoughts.

In addition, as Shi Dingxu (2019) said, if the methods and experiments of neural science can be effectively applied and the processing of middle constructions in the brain can be monitored to find the specific EEG component of the structure processing, such evidence should also promote our understanding of the formation of middle constructions. Or it can be said that the study of generative grammar and the evidence of neural science and technology complete one another.

\section{References}

1. Dai, Manchun. (2001) Syntactic features of English middle constructions. Foreign Language Research, 4, 31-36.

2. Haegeman, L. (1994) Introduction to Government and Binding Theory. Blackwell, Oxford.

3. Han, Jingquan. (2003) Derivation of the English middle constructions. Foreign Language Teaching and Research, $3,179-188$.

4. Han, Jingquan, \& He, Jianzhen. (2004) On Gao Xinggang's analysis of English middle constructions. Journal of PLA University of Foreign Languages, 1, 15-20.

5. He, Xiaowei. (2012) A minimalist approach to the middle construction in English and Chinese. Modern Foreign Language, 1, 14-22.

6. Wang, Heyu, \& Wen, Binli. (2014) A review of syntactic sand semantic research of middle constructions. Modern Foreign Language, 2, 270-278.

7. Wen, Binli. (2002) An Introduction to Syntax. Foreign Language Teaching and Research Press, Beijing.

8. Zhang, Zhiyi, \& Ni, Chuanbin. (2014) The Semantic-syntactic study and classification of English and Chinese middles. Contemporary Foreign Language Research, 10, 5-10. 